\title{
Fractional order polytopic systems: robust stability and stabilisation
}

\author{
Christophe Farges, Jocelyn Sabatier ${ }^{*}$ and Mathieu Moze
}

\author{
* Correspondence: Jocelyn. \\ Sabatier@ims-bordeaux.fr \\ University of Bordeaux, IMS \\ Laboratory (CRONE Team), CNRS \\ UMR 5218, 351 Cours de la \\ Libération, 33405 Talence, France
}

\begin{abstract}
This article addresses the problem of robust pseudo state feedback stabilisation of commensurate fractional order polytopic systems (FOS). In the proposed approach, Linear Matrix Inequalities (LMI) formalism is used to check if the pseudo-state matrix eigenvalues belong to the FOS stability domain whatever the value of the uncertain parameters. The article focuses particularly on the case of a fractional order $v$ such that $0<v<1$, as the stability region is non-convex and associated LMI condition is not as straightforward to obtain as in the case $1<v<2$. In relation to the quadratic stabilisation problem previously addressed by the authors and that involves a single matrix to prove stability of the closed loop system, additional variables are then introduced to decouple system matrices in the closed loop system stability condition. This decoupling allows using parameter-dependent stability matrices and leads to less conservative results as attested by a numerical example.
\end{abstract}

Keywords: Fractional order systems, inear Matrix Inequalities, Robust control, State feedback, Polytopic systems

\section{Introduction}

As for linear time invariant integer order systems, it is now well known that stability of a linear fractional order system depends on the location of the system poles in the complex plane. However, pole location analysis remains a difficult task in the general case. For commensurate fractional order systems, powerful criteria have been proposed. The most well known is Matignon's stability theorem [1]. It permits to check the system stability through the location in the complex plane of the dynamic matrix eigenvalues of the system pseudo-state space representation. Matignon's theorem is in fact the starting point of several results in the field [2,3]. This is the case of the Linear Matrix Inequalities (LMI) stability conditions recently proposed by the authors [4]. These conditions are used to synthesise a stabilising pseudo-state feedback whatever the system fractional order $v$ in the set $] 0,2[$.

Although much progress has been made in the field of fractional system stability, linear time invariant fractional systems robust stability remains an open problem. Among the existing results and only for interval fractional systems, the stability issue was discussed in [5-7]. As commented in [5,8], the result is rather conservative. To reduce the conservatism, in [8], a new robust stability checking method was proposed for interval uncertain systems, where Lyapunov inequality is used for finding the maximum 
eigenvalue of a Hermitian matrix. However, the results presented in [8] only provide sufficient conditions. Note also that these results are dedicated to SISO systems.

In this article, the robust stability and stabilisation problems of linear time invariant fractional order linear systems with convex polytopic uncertainties are studied. The article particularly focuses on the case of a fractional order $v$ such that $0<v<1$, as the stability region is non convex and associated LMI stability condition is not as straightforward to obtain as in the case $1<v<2$. A quadratic stability analysis condition that involves a single matrix variable is proposed in [4]. This condition is used to derive a quadratic pseudo-state feedback synthesis method [4]. In this article, additional variables are introduced to decouple system matrices from the ones proving system stability. This decoupling allows using parameter-dependant stability matrices and lead to less conservative results for both analysis and synthesis purposes as attested by a numerical example.

Notations: The transpose of a matrix $A$ is denoted $A^{\prime}$, its conjugate $\bar{A}$ and its conjugate transpose $A^{*}$. For Hermitian matrices, $>(\geq)$ denotes the Löwner partial order, i.e. $A>B$ iff $A-B$ is (semi) positive definite.

\section{Preliminaries and problem statement}

In this article are considered Linear Time Invariant (LTI) commensurate FOS. In this section, preliminary results are stated in the certain case for an LTI FOS admitting a pseudo-state space representation of the form

$$
\left(\begin{array}{c}
D^{v} x(t) \\
y(t)
\end{array}\right)=\left[\begin{array}{ll}
A & B \\
C & D
\end{array}\right]\left(\begin{array}{l}
x(t) \\
u(t)
\end{array}\right)
$$

where $x(t) \in \mathbf{R}^{n}$ is the pseudo-state vector, $u(t) \in \mathbf{R}^{m}$ is the input vector, $y(t) \in \mathbf{R}^{p}$ is the output vector, $v$ is the fractional order of the system and $A, B, C$ and $D$ are constant matrices. $D^{v}$ is the fractional differentiation operator of order $v$ (presented results are valid whatever definition is used: Riemann-Liouville [9,10] or others [11]). Transfer matrix is $H(s)=C\left(s^{v} I-A\right)^{-1} B+D$ and impulse response matrix is $h(t)=\mathbf{L}^{-1}\{H(s)\}$.

Definition 1 [1] A linear fractional order system defined by its impulse time response $h$ is bounded-input bounded-output (BIBO) stable iff $\forall u \in L^{\infty}\left(\mathbf{R}^{+}, \mathbf{R}^{m}\right), y=$ $h^{*} u \in L^{\infty}\left(\mathbf{R}^{+}, \mathbf{R}^{p}\right)$.

LTI integer order systems stability can be checked via the location of the eigenvalues of the pseudo-state matrix $A$ in the complex plane. This result was extended to LTI commensurate fractional order systems of order $0<v<1$ by Matignon.

Theorem 1 [1] System (1), with minimal triplet $(A, B, C)$ and $0<v<1$, is BIBO stable if and only if

$$
|\operatorname{Arg}(\operatorname{eig}(A))|>v \frac{\pi}{2} \text {. }
$$

This result remains valid when $1<v<2$ as proved in [12]. Stability domain is thus defined as follows:

$$
D s=\left\{z \in \mathrm{C}:|\operatorname{Arg}(z)|>v \frac{\pi}{2}\right\} .
$$

The corresponding stability regions of the complex plane are represented by Figure 1 (grey regions). 

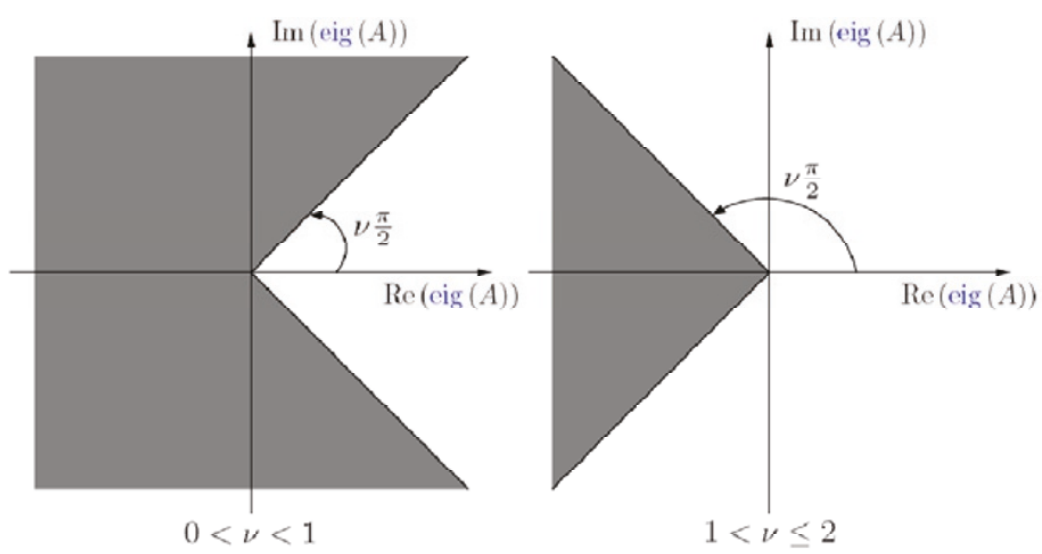

Figure 1 Stability domain of fractional systems (grey region)

Remark 1 Throughout the article, triplet $(A, B, C)$ is always supposed to be minimal.

Testing if the eigenvalues of matrix $A$ belong to a region of the left half plane defined by (3) with $1<v<2$ is a well-known problem in LMI control theory because it corresponds to a performance requirement on the damping ratio of the system. A solution of this problem is provided by the LMI region framework [13]. Extending this LMI condition to the case $0<v<1$ is far from trivial because the location of eigenvalues in this region corresponds to unstable integer order systems. Moreover, region of the complex plane defined by (3) is not convex as shown in Figure 1. However, this problem has been solved in [4] in which the following result was proposed.

Theorem 2 Fractional system (1) of order $0<v<1$ is BIBO stable iff $\exists X=X^{*} \in$ $\mathrm{C}^{n x n}>0$ s.t.

$$
(r X+\bar{r} \bar{X})^{\prime} A^{\prime}+A(r X+\bar{r} \bar{X})<0
$$

$$
\text { where } r=e^{j(1-\nu)} \frac{\pi}{2} \text {. }
$$

Using this result, the pseudo-state feedback stabilisation problem has been solved in [4].

Theorem 3 [4] Fractional system (1) of order $0<v<1$ is BIBO stabilisable by pseudo-state feedback control law $u=K x+y_{r}$ iff $\exists X=X^{*} \in \mathbf{C}^{n x n}>0$ and $Y \in \mathbf{R}^{m \times n}>0$ s.t.

$$
(r X+\bar{r} \bar{X})^{\prime} A^{\prime}+A(r X+\bar{r} \bar{X})+Y^{\prime} B^{\prime}+B Y<0
$$

where $r=e^{j(1-\nu)} \frac{\pi}{2}$. A stabilising controller gain is then:

$$
K=Y(r X+\bar{r} \bar{X})^{-1} .
$$

\section{Feedback stabilisation of polytopic fractional order systems}

\section{Problem statement}

Let the polytopic fractional order system described by:

$$
\left(\begin{array}{c}
D^{v} x(t) \\
y(t)
\end{array}\right)=\left[\begin{array}{ll}
A(\lambda) & B(\lambda) \\
C(\lambda) & D(\lambda)
\end{array}\right]\left(\begin{array}{l}
x(t) \\
u(t)
\end{array}\right)=M(\lambda)\left(\begin{array}{l}
x(t) \\
u(t)
\end{array}\right)
$$


where $\lambda$ is a vector of parametric uncertainties. The parameter-dependent system matrix $M(\lambda)$ belongs to the convex polytope $\mathrm{M}$ with $N$ vertices defined by

$$
M=c o\left\{M_{1}, \cdots, M_{N}\right\}=\left\{M(\lambda)=\sum_{i=1}^{N} \lambda_{i} M_{i}: \lambda \in \Lambda\right\} \text { and } M_{i}=\left[\begin{array}{cc}
A_{i} & B_{i} \\
C_{i} & D_{i}
\end{array}\right]
$$

where $\Lambda=\left\{\lambda \in \mathbf{R}^{N}: \lambda \geq 0, \sum_{i=1}^{N} \lambda_{i}=1\right\}$.

This article is devoted to giving constructive conditions for pseudo-state feedback control laws of the form $u=K x+y_{\mathrm{r}}$, where $K$ is a constant matrix gain and $y_{\mathrm{r}}$ is the reference signal, robustly stabilising the closed loop system:

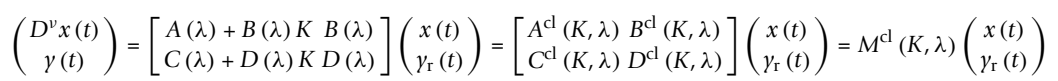

The closed loop system matrices $M^{\mathrm{cl}}(\lambda)$ belong to the polytope $\mathrm{M}^{\mathrm{cl}}$ defined by:

$$
\mathrm{M}^{\mathrm{cl}}=c o\left\{M_{1}^{\mathrm{cl}}, \ldots M_{N}^{\mathrm{cl}}\right\}
$$

where

$$
M_{i}^{\mathrm{cl}}=\left[\begin{array}{cc}
A_{i}+B_{i} K & B_{i} \\
C_{i}+D_{i} K & D_{i}
\end{array}\right]=\left[\begin{array}{cc}
A_{i}^{\mathrm{cl}} & B_{i}^{\mathrm{cl}} \\
C_{i}^{\mathrm{cl}} & D_{i}^{\mathrm{cl}}
\end{array}\right]
$$

The next sections present two results on robust control of MIMO fractional order systems. The first one is a straightforward extension of Theorem 3 to handle uncertain polytopic fractional systems (7). In the second one, elimination lemma is used to derive a less conservative condition.

\section{Polytopic stabilisation}

Using a single matrix $X$ to attest stability for the whole set of uncertainty is known to be overly conservative. However, the coupling between stability matrix $X$ and dynamic matrix $A(\lambda)$ prevents from directly using a parameter-dependent stability matrix $X_{\mathrm{cl}}$ $(\lambda)$. The following result allows us to overcome this problem.

Theorem 4 Fractional polytopic system (7) of order $0<v<1$ is robustly BIBO stable if there exist $N$ matrices $X_{i}=X_{i}^{*} L C^{n \times n}, X_{i}>0$ and a matrix $G L C^{2 n \times n}$ s.t. $\forall i L\{1 . N\}$ :

$$
\left[\begin{array}{cc}
(0) & \left(r X_{i}+\bar{r} \bar{X}_{i}\right)^{\prime} \\
\left(r X_{i}+\bar{r} \bar{X}_{i}\right) & (0)
\end{array}\right]+\left[\begin{array}{c}
A_{i} \\
-I
\end{array}\right] G^{\prime}+G\left[A_{i}^{\prime}-I\right]<0 .
$$

Proof Suppose a solution $\left(X_{i}, G\right)$ to (11). Computation of convex combination over the $N$ vertices allows to write for all uncertainties:

$$
\left[\begin{array}{cc}
(0) & (r X(\lambda)+\bar{r} \bar{X}(\lambda))^{\prime} \\
(r X(\lambda)+\bar{r} \bar{X}(\lambda)) & (0)
\end{array}\right]+\left[\begin{array}{c}
A(\lambda) \\
-I
\end{array}\right] G^{\prime}+G\left[A^{\prime}(\lambda)-I\right]<0
$$

with

$$
X(\lambda)=\sum_{i=1}^{N} \lambda_{i} X_{i}
$$


Applying elimination lemma [14] to the last inequality leads to:

$$
\left[I_{n} A(\lambda)\right]\left[\begin{array}{cc}
0 & (r X(\lambda)+\bar{r} \bar{X}(\lambda))^{\prime} \\
r X(\lambda)+\bar{r} \bar{X}(\lambda) & 0
\end{array}\right]\left[\begin{array}{c}
I_{n} \\
A^{\prime}(\lambda)
\end{array}\right]<0
$$

which is exactly (4) with parameter-dependant matrices and thus, according to Theorem 2, proves the robust stability of the system.

Theorem 4 provides a sufficient condition for stability but decoupling between stability matrix and dynamic matrix in (12) allows to use the parameter-dependant stability matrix $X(\lambda)$ defined by (13). As proved in $[15,16]$, stability condition of Theorem 4 is always less conservative than the one of theorem 18 in [4] based on the use of quadratic stability condition.

Based on Theorem 4, the following result allows to design a pseudo state feedback control law while stability of the closed loop system is attested by a parameter-dependant stability matrix.

Theorem 5 Fractional polytopic system (7) of order $0<v<1$ is robustly BIBO stabilisable by pseudo-state feedback control law $u=K x+y_{r}$ if there exist $N$ matrices $X_{i}=$ $X_{i}^{*} L C^{n \times n}, X_{i}>0, F L R^{n \times n}$ and $K_{t} L R^{m \times n}$ s.t. $\forall i L\{1 . N\}$ :

$$
\left[\begin{array}{cc}
(0) & \left(r X_{i}+\bar{r} \bar{X}_{i}\right)^{\prime} \\
\left(r X_{i}+\bar{r} \bar{X}_{i}\right) & (0)
\end{array}\right]+\left[\begin{array}{c}
A_{i} F+B_{i} K_{t} \\
-F
\end{array}\right]\left[A_{0}^{\prime}-I_{m}\right]+\left[\begin{array}{c}
A_{0} \\
-I_{m}
\end{array}\right]\left[\left(A_{i} F+B_{i} K_{t}\right)^{\prime}-F^{\prime}\right]<0 .
$$

A stabilising controller gain is then:

$$
K=K_{t} F^{-1} \text {. }
$$

Proof Suppose a solution $\left(X_{i}, F, K_{t}\right)$ to (15). Then the controller definition implies that $K_{t}=K F$, which allows to write:

$$
\left[\begin{array}{cc}
(0) & \left(r X_{i}+\bar{r} \bar{X}_{i}\right)^{\prime} \\
\left(r X_{i}+\bar{r} \bar{X}_{i}\right) & (0)
\end{array}\right]+\left[\begin{array}{c}
A_{i}+B_{i} K \\
-I
\end{array}\right] F\left[A_{0}{ }^{\prime}-I\right]+\left[\begin{array}{c}
A_{0} \\
-I
\end{array}\right] F^{\prime}\left[\left(A_{i}+B_{i} K\right)^{\prime}-I\right]<0 .
$$

Defining $G=\left[\begin{array}{c}A_{0} \\ -I\end{array}\right] F^{\prime}$ gives

$$
\left[\begin{array}{cc}
(0) & \left(r X_{i}+\bar{r} \bar{X}_{i}\right)^{\prime} \\
\left(r X_{i}+\bar{r} \bar{X}_{i}\right) & (0)
\end{array}\right]+\left[\begin{array}{c}
A_{i}+B_{i} K \\
-I
\end{array}\right] G^{\prime}+G\left[\left(A_{i}+B_{i} K\right)^{\prime}-I\right]<0 .
$$

According to Theorem 4, this last inequality proves that the closed loop system is robustly stable.

In order to maintain convexity, matrix $A_{0}$ appearing in Theorem 5 cannot be a variable but must be chosen a priori such that it is stable. Indeed, elimination lemma shows that existence of matrices $X_{i}=X_{i}^{*}>0, F$ and $A_{0}$ verifying (15) is equivalent to existence of matrices $X_{i}=X_{i}^{*}>0$ and $A_{0}$ verifying the following two inequalities:

$$
\begin{aligned}
& {\left[\begin{array}{ll}
I & A_{i}
\end{array}\right]\left[\begin{array}{cc}
0 & \left(r X_{i}+\bar{r} \bar{X}_{i}\right)^{\prime} \\
r X_{i}+\bar{r} \bar{X}_{i} & 0
\end{array}\right]\left[\begin{array}{c}
1 \\
A_{i}^{\prime}
\end{array}\right]<0,} \\
& {\left[\begin{array}{ll}
I & A_{0}
\end{array}\right]\left[\begin{array}{cc}
0 & \left(r X_{i}+\bar{r} \bar{X}_{i}\right)^{\prime} \\
r X_{i}+\bar{r} \bar{X}_{i} & 0
\end{array}\right]\left[\begin{array}{c}
1 \\
A_{0}^{\prime}
\end{array}\right]<0 .}
\end{aligned}
$$


Remark Contrary to the analysis case, synthesis result of Theorem 5 cannot be proved to be always less conservative than the one of Theorem 18 in [4] based on the use of quadratic stability condition. However, improvement can be significant on some given examples, as shown in next section.

\section{Numerical example}

The proposed numerical application is a fractional version of an example proposed in [17]. Studied system is described by representation (7) where:

$$
A=\left[\begin{array}{cc}
-3+\alpha & 2 \\
-3 & 1.5+\gamma
\end{array}\right] \quad B=\left[\begin{array}{l}
1 \\
\beta
\end{array}\right]
$$

with

$$
|\alpha| \leq \alpha_{\max } \quad|\beta| \leq 0.5 \quad|\gamma| \leq 1.5
$$

Fractional order $v$ is chosen equal to 0.7. As parameters $\alpha, \beta$ and $\gamma$ vary in the intervals defined by relation (22), eigenvalues of matrix $A$ are represented Figure 2 for $\alpha_{\max }$ $=0.7$. That figure demonstrates that the system can be stable or unstable depending on the values $\alpha, \beta$ and $\gamma$ uncertain parameters values.

The goal is now to compute a pseudo-state feedback control law of the form $u=K x$ $+y_{\mathrm{r}}$ that robustly stabilises the system.

For comparison purpose, quadratic stabilisation theorem proposed in [4] and theorem proposed in section "Feedback stabilisation of polytopic fractional order systems" are applied to compute a stabilising pseudo-state feedback for model (21-22).

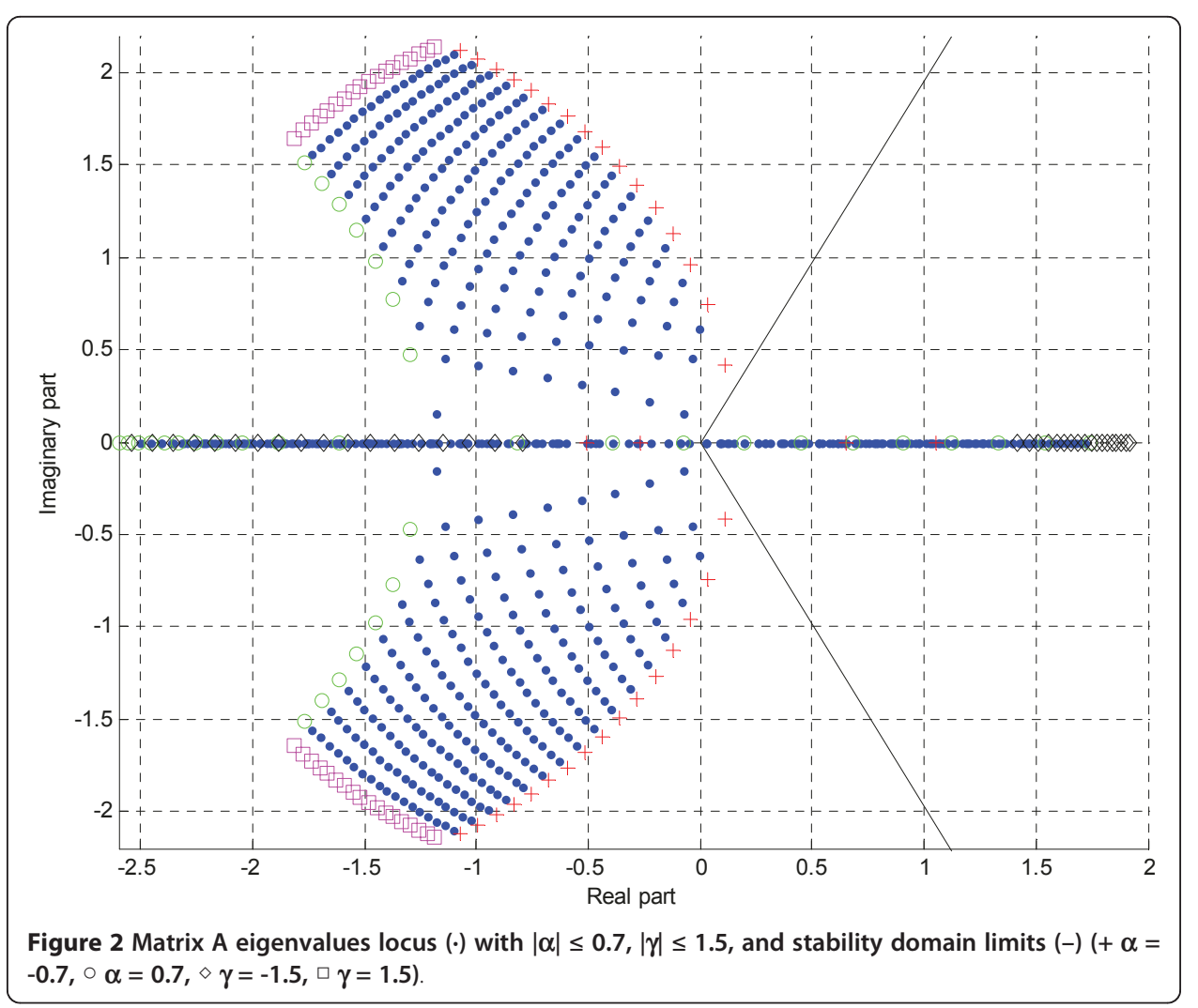


First, Theorem 18 in [4] is used to compute a stabilising controller of gain $K$. Parser Yalmip [18] and LMI solver SDPT3 are used to get matrices $X$ and $Y$ solutions of the semi-definite problem associated with LMI condition

$$
(r X+\bar{r} \bar{X})^{\prime} A_{i}^{\prime}+A_{i}(r X+\bar{r} \bar{X})+Y^{\prime} B_{i}^{\prime}+B_{i} Y<0
$$

where $r=e^{j(1-v)} \frac{\pi}{2}, \times=X^{*} \in C^{\mathrm{n} \times \mathrm{n}}, X>0$ and $Y \in \mathrm{R}^{\mathrm{m} \times \mathrm{n}}$ s.t. $\forall i \in\{1 . N\}$. A stabilising controller gain is then:

$$
K=Y(r X+\bar{r} \bar{X})^{-1}
$$

For such a problem, a solution exists for values of $\alpha_{\max }$ up to $\alpha_{\max }^{\text {quad }}=0.57$.

Then, this solver has been used to get matrices $X_{i}, i \in\{1 . N\}, F$ and $K_{t}$ associated with LMI condition (15) of Theorem 5 with the matrix $A_{0}$ chosen equal to:

$$
A_{0}=\left[\begin{array}{cc}
-2 & 0 \\
0 & -2
\end{array}\right] \text {. }
$$

A solution exists for values of $\alpha_{\max }$ up to $\alpha_{\max }^{A_{0}}=0.74$ and corresponding gain $K$ is obtained using Equation 14:

$$
K=\left[\begin{array}{ll}
-15.56 & 33.29
\end{array}\right] \text {. }
$$

This represents an improvement of about $30 \%$.

Eigenvalues of matrix $A+B K$ (closed loop state matrix) as parameters $\alpha, \beta$ and $\gamma$ vary in the intervals defined by relation (24) with $\alpha_{\max }=0.74$ are represented Figure 3 . This figure confirms that the closed loop system is robustly BIBO stable.

The degrees of freedom offered by matrix $A_{0}$ are now used to find a controller $K$ for higher values of parameter $\alpha_{\max }$. Matrix $A_{0}$ is first chosen equal to:

$$
A_{0}^{1}=\left[\begin{array}{cc}
\lambda_{r} & -\lambda_{i} \\
\lambda_{i} & \lambda_{r}
\end{array}\right]
$$

Figure 4 represents the $\alpha_{\max }$ values obtained with $-50 \leq \lambda_{r} \leq 0$ and $0 \leq \lambda_{i} \leq 2$. This figure highlights the existence of an infinity of matrices $A_{0}$ that permit to obtain a solution and provides a value of $\alpha_{\max }$ equal to $\alpha_{\max }^{A_{0}^{1}}=0.86$ for $\lambda_{r}=-15.86$ and $\lambda_{i}=0$.

Matrix $A_{0}$ is now chosen equal to:

$$
A_{0}^{2}=\left[\begin{array}{cc}
\lambda_{r 1} & 0 \\
0 & \lambda_{r 2}
\end{array}\right]
$$

Figure 5 represents the $\alpha_{\max }$ values obtained with $-50 \leq \lambda_{r_{1}} \leq 0$ and $0 \leq \lambda_{r_{2}} \leq 2$. As previously, this figure shows that the maximal value of $\alpha_{\max }\left(\alpha_{\max }^{A_{0}^{2}}=0.88\right)$ is obtained for $A_{0}$ eigenvalues $\lambda_{r_{1}}=-19.31$ and $\lambda_{r_{2}}=-19.31$.

As shown in Table 1 , the result provided by Theorem 5 , substantially increases the size of the uncertain domain for which a controller can be computed. The best obtained $\alpha_{\max }$ is 0.88 and corresponds to an improvement of $54 \%$ over the quadratic case. 


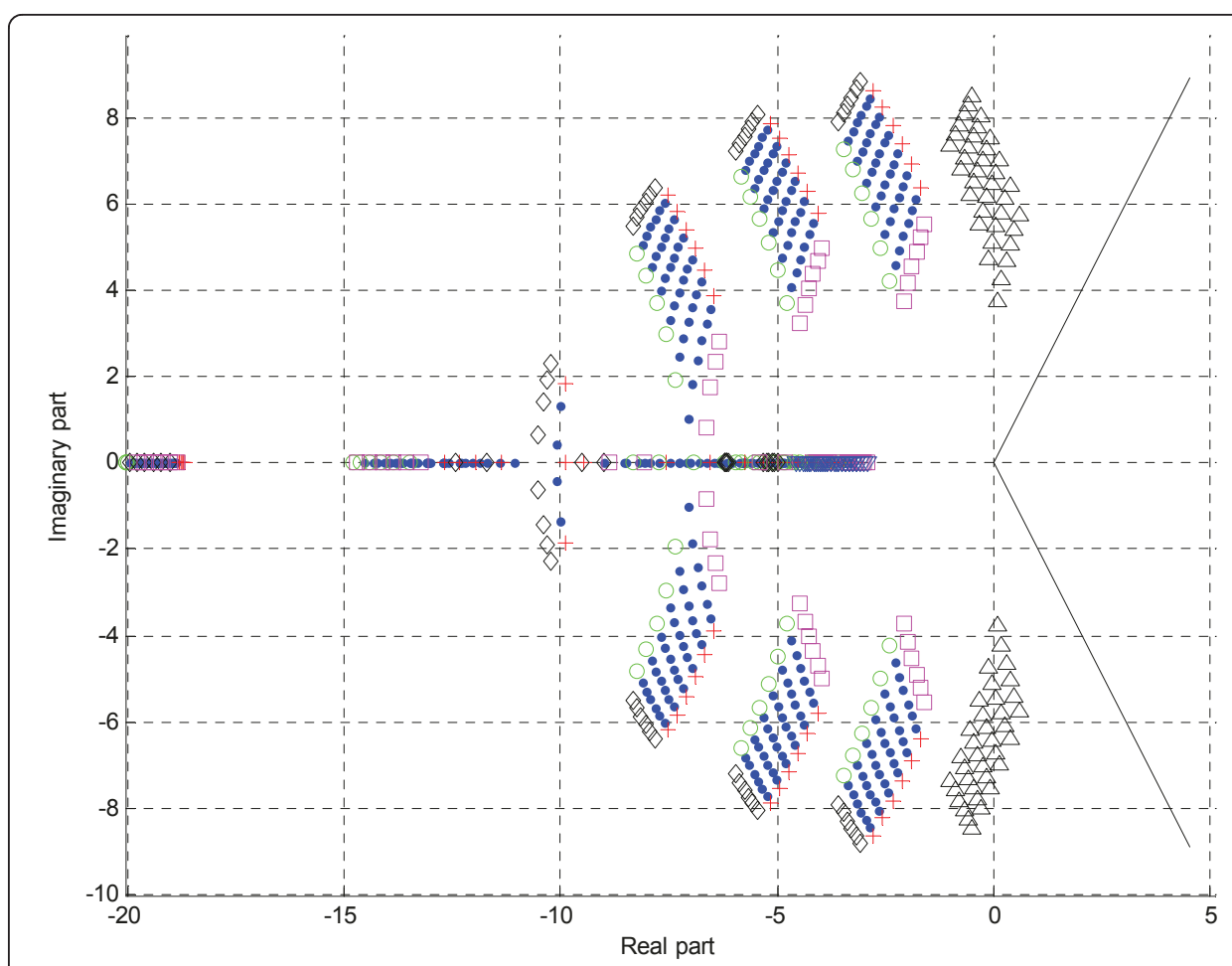

Figure 3 Matrix $A+B K$ eigenvalues locus (.) with $|\alpha| \leq 0.7,|\gamma| \leq 1.5,|\beta| \leq 0.5$, stability domain limits $(-)(+\alpha=-0.7, \circ \alpha=0.7, \diamond \gamma=-1.5, \square \gamma=1.5, \nabla \beta=-0.5, \Delta \beta=0.5)$.

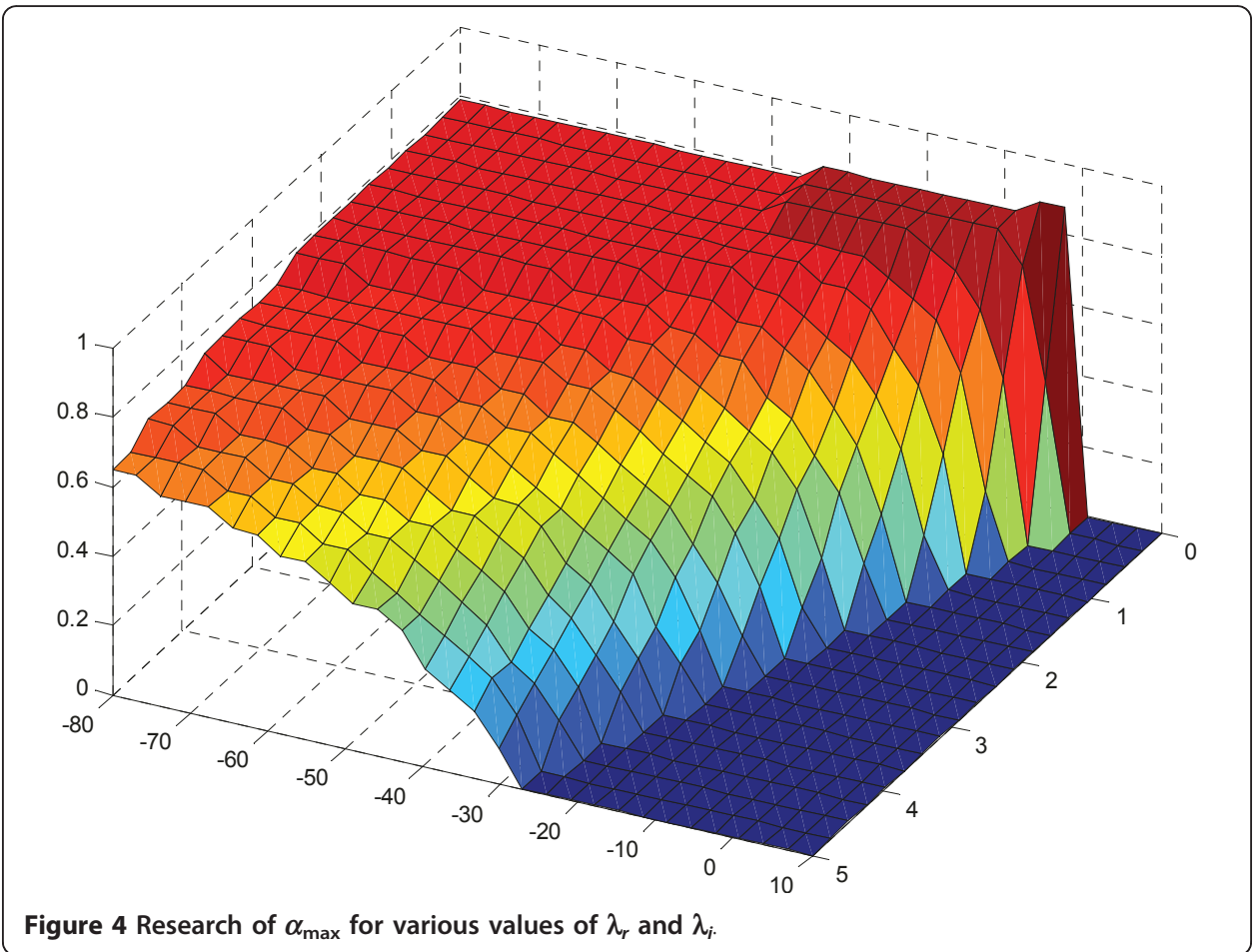




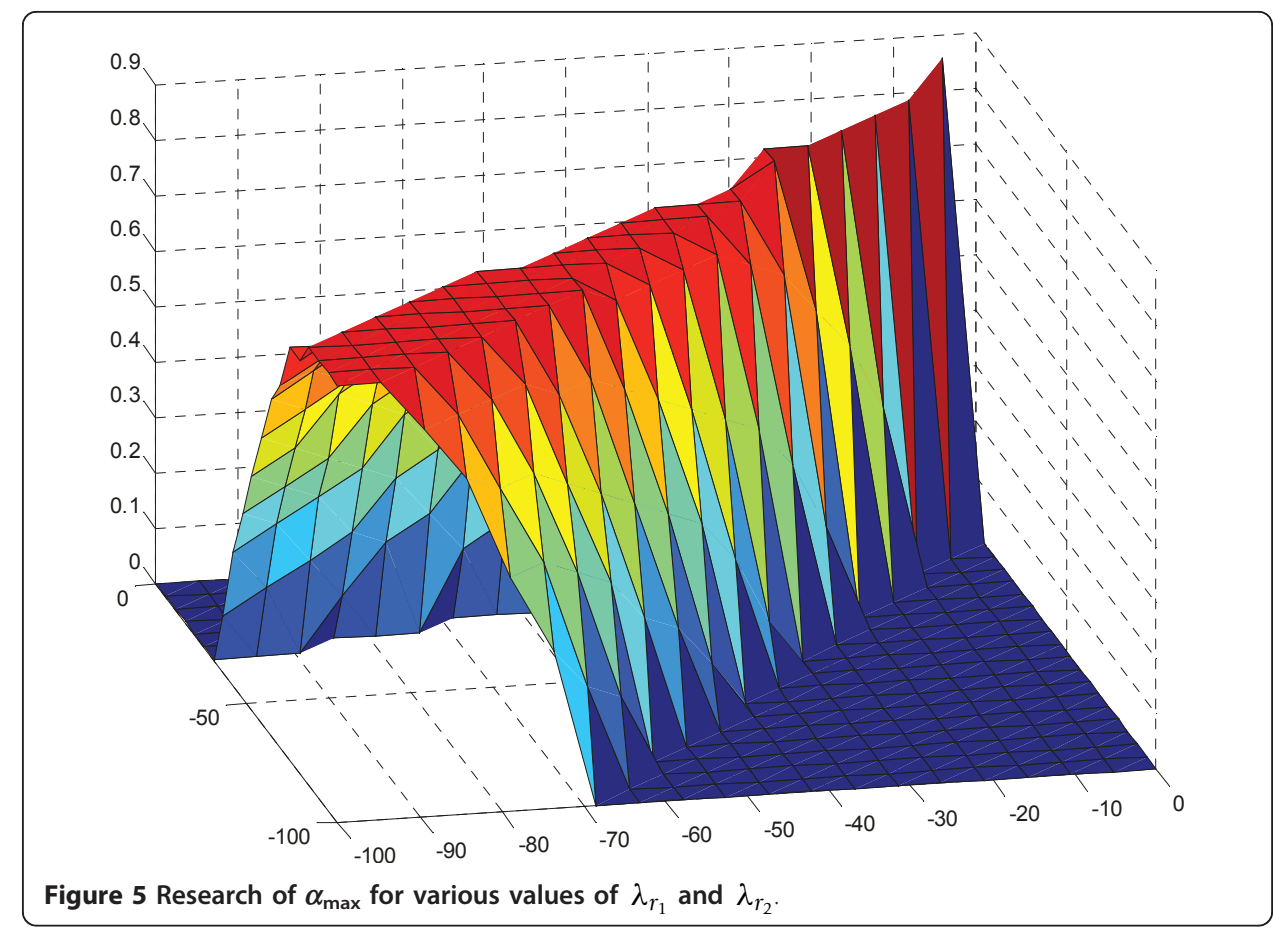

\section{Conclusion}

In this article, a solution is proposed for the robust stability and stabilisation problems of fractional order linear systems subjected to convex polytopic uncertainties. Presented results are derived from the LMI stability analysis and synthesis conditions recently proposed by the authors for the certain case [4].

In relation to the analysis result proposed in [4] that involves a single matrix in order to prove stability of the system, additional variables are then introduced to decouple system matrices from the ones proving stability of the closed loop system. This decoupling allows using parameter-dependant stability matrices and obtained LMI stability analysis condition is always less conservative than the one involving a single stability matrix.

The method is extended to handle the state feedback synthesis problem. Although, synthesis result based on the use of parameter-dependant matrices cannot be proved to be always less conservative than the quadratic one, significant improvement is obtained on a numerical example.

As shown in the numerical example, this last condition offers some degree of freedom. Some parameters have to be set a priori and this choice has an influence on the quality of the obtained result. Authors are currently working on a systematic method to choose those parameters.

Table 1 Values of $\alpha_{\max }$ obtained

\begin{tabular}{cccc}
\hline$\alpha_{\max }^{\text {quad }}$ & $\alpha_{\max }^{A_{0}}$ & $\alpha_{\max }^{A_{0}^{1}}$ & $\alpha_{\max }^{A_{0}^{2}}$ \\
\hline 0.57 & 0.74 & 0.86 & 0.88 \\
\hline
\end{tabular}


Abbreviations

BIBO: bounded-input bounded-output; FOS: fractional order polytopic systems; LMI: Linear Matrix Inequalities; LTI: Linear Time Invariant.

\section{Authors' contributions}

All the authors have contributed in all the paper part.

\section{Competing interests}

The authors declare that they have no competing interests.

Received: 11 December 2010 Accepted: 22 September 2011 Published: 22 September 2011

\section{References}

1. Matignon, D: Stability results on fractional differential equations with applications to control processing. pp. 963-968. Computational Engineering in Systems Applications, Lille, France2, (1996)

2. Moze, M, Sabatier, J, Oustaloup, A: LMI tools for stability analysis of fractional systems. 20th ASME International Design Engineering Technical Conferences \& Computers and Information in Engineering Technical Conference (ASME IDETC/ CIE'05), Long Beach, California, USA (2005)

3. Sabatier, J, Farges, C: On stability of fractional order systems. International Journal of Bifurcation and Chaos. (2011, in press)

4. Farges, C, Moze, M, Sabatier, J: Pseudo state feedback stabilization of commensurate fractional order systems. Automatica. 46(10), 1730-1734 (2010). doi:10.1016/j.automatica.2010.06.038

5. Chen, YQ, Ahn, HS, Podlubny, I: Robust stability check of fractional order linear time invariant systems with interval uncertainties. Signal Process. 86, 2611-2618 (2006). doi:10.1016/j.sigpro.2006.02.011

6. Petras, I, Chen, Y, Vinagre, BM, Podlubny, I: Stability of linear time invariant systems with interval fractional orders and interval coefficients. pp. 1-4. Proceedings of the International Conference on Computation Cybernetics (ICCC04), Vienna, Austria (2004)

7. Tan, N, Ozguven, OF, Ozyetkin, MM: Robust stability analysis of fractional order interval polynomials. ISA Trans. 48(2), 166-172 (2009). doi:10.1016/j.isatra.2009.01.002

8. Ahn, HS, Chen, YQ, Podlubny, I: Robust stability test of a class of linear time-invariant interval fractional-order system using Lyapunov inequality. Appl Math Comput. 187(1), 27-34 (2007). doi:10.1016/j.amc.2006.08.099

9. Caputo, M: Linear models of dissipation whose q is almost frequency independent-II. Geophys. J Int. 13(5), 529-539 (1967)

10. Miller, KS, Ross, B: An Introduction to the Fractional Calculus and Fractional Differential Equations. Wiley, New York (1993)

11. Samko, SG, Kilbas, AA, Marichev, Ol: Fractional Integrals and Derivatives: Theory and Applications. Gordon and Breach Science Publishers, Amsterdam (1993)

12. Sabatier, J, Moze, M, Farges, C: LMI Stability conditions for fractional order systems. Comput Math Appl. 9, 1594-1609 (2010)

13. Boyd, S, El Ghaoui, L, Feron, E, Balakrishnan, V: Linear Matrix Inequalities in System and Control Theory. SIAM Studies in Applied Mathematics, Philadelphia. (1994)

14. Skelton, RE, Iwazaki, T, Grigoriadis, K: A Unified Approach to Linear Control Design. Taylor and Francis, London (1998)

15. Paucelle, D: Formulation générique de problèmes en analyse et commande robuste par les fonctions de Lyapunov dépendant des paramètres. PhD thesis dissertation, Toulouse III University (2000)

16. Peaucelle, D, Arzelier, D: New LMI-based conditions for robust $\mathrm{H} 2$ performance analysis. Proceedings of the American Control Conference, Chicago Illinois. 317-321 (2000)

17. De Souza, CE, Trofino, A: An LMl approach to stabilization of linear discrete-time periodic systems. Int J Control. 73, 696-709 (2000). doi:10.1080/002071700403466

18. Lofberg, J: A toolbox for modeling and optimization in MATLAB. Proceedings of the CACSD Conference, Taipei, Taiwan (2004)

doi:10.1186/1687-1847-2011-35

Cite this article as: Farges et al:: Fractional order polytopic systems: robust stability and stabilisation. Advances in Difference Equations 2011 2011:35.

\section{Submit your manuscript to a SpringerOpen ${ }^{\circ}$ journal and benefit from:}

- Convenient online submission

Rigorous peer review

- Immediate publication on acceptance

- Open access: articles freely available online

- High visibility within the field

- Retaining the copyright to your article

Submit your next manuscript at $\$$ springeropen.com 